\title{
Four- and Five-Coordinate Aluminum Ketiminate Complexes: Synthesis, Characterization, and Ring-Opening Polymerization
}

\author{
Ru-Ching Yu, ${ }^{\dagger}$ Chen-Hsiung Hung, ${ }^{\dagger}$ Jui-Hsien Huang, ${ }^{,}, \dagger$ Horng-Yi Lee, ${ }^{\ddagger}$ and Jwu-Ting Chen ${ }^{\ddagger}$ \\ Department of Chemistry, National Changhua University of Education, Changhua, Taiwan 500, \\ and Department of Chemistry, National Taiwan University, Taipei, Taiwan 106
}

Received June 10, 2002

\begin{abstract}
A series of aluminum complexes featuring with the ketiminate ligand, OCMeCHCMeNHAr $\left(\mathrm{Ar}=2,6-\mathrm{Pr}_{2} \mathrm{C}_{6} \mathrm{H}_{3}, 1\right)$, have been prepared and characterized spectroscopically and structurally. Reactions of 1 with trialkylaluminum in 1:1 or 1:2 molar ratio generate four- and five-coordinated aluminum complexes (OCMeCHCMeNAr)AIR $(R=\mathrm{Me}$ (2); $\mathrm{R}=\mathrm{Et}(3))$ and $(\mathrm{OCMeCHCMeNAr})_{2} \mathrm{AIR}(\mathrm{R}=\mathrm{Me}(4) ; \mathrm{R}=\mathrm{Et}(5))$ in high yields. Similarly, reaction of $\mathrm{AlCl}_{3}$ with 1 or 2 equiv of the lithiated 1 in toluene afforded bis(ketiminate) aluminum chloride complex, (OCMeCHCMeNAr) ${ }_{2} \mathrm{AICl}$ (6) or $(\mathrm{OCMeCHCMeNAr}) \mathrm{AlCl}_{2}$ (7). Surprisingly, reacting 6 with 1 equiv of $\mathrm{AgBF}_{4}$ in methylene/acetonitrile mixsolvents generates $(\mathrm{OCMeCHCMeNAr})_{2} \mathrm{AIF}(8)$ in moderate yield. The structures of complexes 2-6 and 8 have been determined by X-ray crystallography. Complexes 2 and 3 both exhibit tetrahedron structures with the aluminum atom surrounded by oxygen and nitrogen atoms of chelating ketiminate and two alkyl groups. The mono- and bis-ketiminate aluminum complexes $\mathbf{2}-\mathbf{5}$ have shown moderate activity toward the ring-opening polymerization of $\epsilon$-caprolactone.
\end{abstract}

\section{Introduction}

The aluminum complexes draw considerable attention for organic synthesis ${ }^{1}$ and polymerization ${ }^{2}$ due to their strong Lewis acidity and inexpensiveness. In addition to the aluminum atom itself, the surrounded coordinating ligands affect the properties of the aluminum complexes sterically and electronically. $\beta$-Diketiminate ${ }^{3}$ and Schiff base (Chart $1)$, monoanionic bidentate ligands, are used extensively in synthesizing organometallic complexes. ${ }^{4}$ More recently, the $\beta$-diketiminate and Schiff base, with bulky substitutents, have raised much attention because they are able to stabilize metal complexes $^{5}$ and to polymerize cyclic monomer.

In viewing the coordinating sphere of the metal complexes, steric bulky ligands can stabilize catalysts; however, con-

* E-mail: juihuang@cc.ncue.edu.tw.

National Changhua University of Education.

$\div$ National Taiwan University.

(1) Yamamoto, H., Ed. Lewis Acids in Organic Synthesis; Wiley-VCH: New York, 2000.

(2) Bochmann, M.; Dawson, D. M. Angew. Chem., Int. Ed. Eng. 1996, 35, 2226. (b) Atwood, D. A.; Jegier, J. A.; Rutherford, D. J. Am. Chem. Soc. 1995, 117, 6779. (c) Coles, M. P.; Jordan, R. F. J. Am. Chem. Soc. 1997, 119, 8125 .

(3) Parks, J. E.; Holm, R. H. Inorg. Chem. 1968, 7, 1408. (b) Hitchcock, P. B.; Lappert, M. F.; Layh, M. Chem. Commun. 1998, 201. (c) Clegg, W.; Cope, E. K.; Edwards, A. J.; Mair, F. S. Inorg. Chem. 1998, 37, 2317.

6450 Inorganic Chemistry, Vol. 41, No. 24, 2002
Chart 1

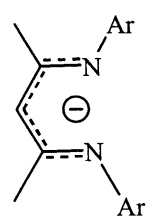

$\beta$-diketiminate

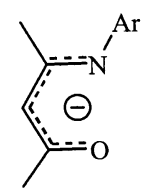

ketiminate

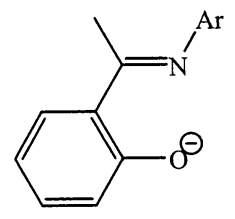

Schiff-base strained ligands leave the catalysts more open toward the entering monomer. To obtain stable aluminum complexes as well as good activity toward monomer, we have decided to investigate the aluminum complexes containing ketiminate ligand. Few studies have involved the aluminum complexes with mono anionic bidentate ketiminate ligands (Chart 1).

(4) Qian, B.; Scanlon, W. J., IV; Smith, M. R., III. Organometallics 1999 18, 1693. (b) Qian, B.; Ward, D. L.; Smith, M. R., III. Organometallics 1998, 17, 3070. (c) Rahim, M.; Taylor, N. J.; Xin, S.; Collins, S. Organometallics 1998, 17, 1315. (d) Kakaliou, L.; Scanlon, W. J., IV.; Qian, B.; Baek, S. W.; Smith, M. R., III. Inorg. Chem. 1999, 38, 5964. (e) Vollmerhaus, R.; Rahim, M.; Tomaszewski, R.; Xin, S.; Taylor, N. J.; Collins, S. Organometallics 2000, 19, 2161. (f) Hitchcock, P. B.; Lappert, M. F.; Liu, D.-S. J. Chem. Soc. Chem. Commun. 1994, 1699. (g) Hitchcock, P. B.; Lappert, M. F.; Liu, D.S. J. Chem. Soc. Chem. Commun. 1994, 2637. (h) Budzelaar, P. H. M.; Moonen, N. N. P.; de Gelder, R.; Smits, J. M. M.; Gal, A. W. Eur. J. Inorg. Chem. 2000, 753. 
Scheme 1<smiles></smiles>

(7)<smiles>CC1=CC(C)=N[Tl]O1</smiles>

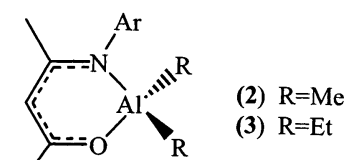

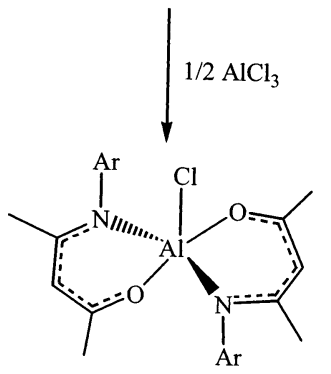

(6)<smiles>CC1=CC(C)=NOCN1[AlH2]</smiles>

(1); $\mathrm{Ar}=2,6-{ }^{\mathrm{i}} \mathrm{Pr}_{2} \mathrm{C}_{6} \mathrm{H}_{3}$ $1 / 2 \mathrm{AlR}_{3}$

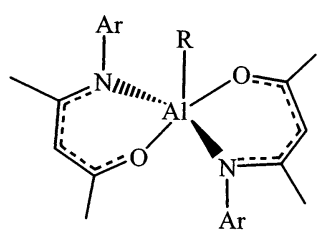

(4) $\mathrm{R}=\mathrm{Me}$ (5) $\mathrm{R}=\mathrm{Et}$
Bidentate monoanionic ketiminate ligands bind to metals that can result in a six-membered ring and cause the metal atoms surrounded by bulky substitutents on one side but left the other side open. Here we report the syntheses and characterization of aluminum complexes and their reactivity toward $\epsilon$-caprolactone.

\section{Results and Discussion}

Synthesis and Characterization. Ketimine (1) can be prepared easily according to the published procedure ${ }^{3 a}$ by reacting a 1:1 ratio of 2,4-pentandione and 2,6-diisopropylaniline in methanol with a small amount of formic acid as catalysts. Reactions of ketimine $1\left(\mathrm{Ar}=2,6-{ }^{i} \operatorname{Pr}_{2} \mathrm{C}_{6} \mathrm{H}_{3}\right)$ with trialkylaluminum in 1:1 molar ratio generate four-coordinated aluminum complexes (OCMeCHCMeNAr)AlR $2(\mathrm{R}=\mathrm{Me}$ (2); $\mathrm{R}=\mathrm{Et}(\mathbf{3})$ ) in high yield (Scheme 1). The reactions proceed along with the elimination of 1 equiv of methane or ethane. Similarly, while reacting $\mathbf{1}$ and trialkylaluminum

(5) Feldman, J.; McLain, S. J.; Parthasarathy, A.; Marshall, W. J.; Calabrese, J. C.; Arthur, S. O. Organometallics 1997, 16, 1514. (b) Hardman, N. J.; Power, P. P. Inorg. Chem. 2001, 40, 2474. (c) Stender, M.; Eichler, B. E.; Hardman, N. J.; Power, P. P.; Prust, J.; Noltemeyer, M.; Roesky, H. W. Inorg. Chem. 2001, 40, 2794. (d) Radzewich, C. E.; Guzei, I. A.; Jordan, R. F. J. Am. Chem. Soc. 1999, 121, 8673. (e) Gibson, V. C.; Segal, J. A.; White, A. J. P.; Williams, D. J. J. Am. Chem. Soc. 2000, 122, 7120. (f) Radzewich, C. E.; Coles, M. P.; Jordan, R. F. J. Am. Chem. Soc. 1998, 120, 9384. (g) Cui, C.; Roesky, H. W.; Hao, H.; Schmidt, H.-G.; Noltemeyer, M. Angew. Chem., Int. Ed. 2000, 39, 1815. (h) Cui, C.; Roesky, H. W.; Schmidt, H.-G.; Noltemeyer, M.; Hao, H.; Cimpoesu, F. Angew. Chem., Int. Ed. 2000 39, 4274. (i) Gibson, V. C.; Maddox, P. J.; Newton, C.; Redshaw, C.; Solan, G. A.; White, A. J. P.; Williams, D. J. Chem. Commun. 1998 1651. (j) Lee, L. W. M.; Piers, W. E.; Elsegood, M. R. J.; Clegg, W.; Parvez, M. Organometallics 1998, 18, 2947. (k) Cheng, M.; Lobkovsky, E. B.; Coates, G. W. J. Am. Chem. Soc. 1998, 120, 11018. (1) Cheng, M.; Attygalle, A. B.; Lobkovsky, E. B.; Coates, G. W. J. Am. Chem. Soc. 1999, 121, 11583.
Scheme 2

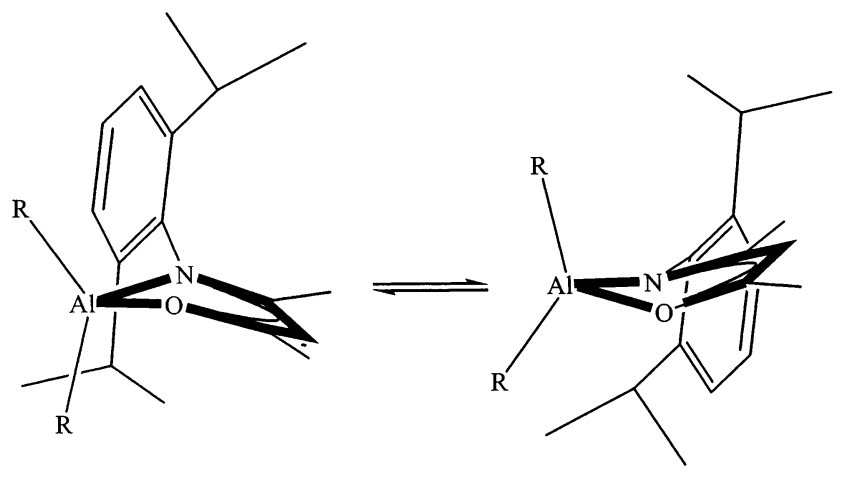

in 1:2 ratio, bis(ketiminate) aluminum complexes, $(\mathrm{OCMeCH}-$ CMeNAr $)_{2} \mathrm{AlR}(\mathrm{R}=\mathrm{Me}(\mathbf{4}) ; \mathrm{R}=\mathrm{Et}(\mathbf{5}))$, were obtained. However, complexes $\mathbf{4}$ and $\mathbf{5}$ are more sterically hindered and hence more strict conditions, i.e., refluxing for 3 days in toluene, are needed in order to obtain reasonable yield.

All the compounds have been characterized by ${ }^{1} \mathrm{H}$ and ${ }^{13} \mathrm{C}$ NMR spectra, where the proton and carbon of the methine groups of the backbone serve as excellent indicators for evaluating the purity of the metal complexes. All the complexes 2-5 show one resonance for the methine of the backbone at ca. $\delta 5.0$ and ca. $\delta 100.0$ for ${ }^{1} \mathrm{H}$ and ${ }^{13} \mathrm{C} \mathrm{NMR}$ spectroscopy, respectively. For the mono-ketiminate compounds $\mathbf{2}$ and $\mathbf{3}$, one isopropyl methine resonance and two isopropyl methyl resonances were observed. This is consistent with fast ring inversion, as shown in Scheme 2, and slow aryl rotation. For the bis-ketiminate complexes 4-6, the two ketiminate ligands are equivalent but the two isopropyl groups on a given ketiminate are inequivalent and the two methyl groups within each isopropyl group are inequivalent. Therefore four methyl and two methine resonances for the isopropyl groups are expected for the slow ring inversion and slow aryl rotation. However, the ${ }^{1} \mathrm{H}$ NMR spectra of 4-6 are all shown one methyl resonance for the isopropyl groups; that is no surprise due to the overlapping of the methyl groups. By carefully reviewing the ${ }^{13} \mathrm{C}\left\{{ }^{1} \mathrm{H}\right\}$ NMR data, all the methyl resonances of the isopropyl groups in complexes 4-6 can be resolved.

Reactions of $\mathrm{AlCl}_{3}$ with 1 or 2 equiv of the lithiated ketimine $\mathbf{1}$, obtained from the reaction of ketimine $\mathbf{1}$ with 2.5 $\mathrm{M} n$-BuLi in toluene, afforded bis(ketiminate) aluminum chloride complex, (OCMeCHCMeNAr) $)_{2} \mathrm{AlCl}$ (6) or (OC$\mathrm{MeCHCMeNAr} \mathrm{AlCl}_{2}$ (7) in high yield (Scheme 1). The methine proton of the backbone of complexes 6 and 7 exhibits one resonance at ca. $\delta 5.2$ for the ${ }^{1} \mathrm{H}$ NMR spectroscopy. Again, the methine protons of the isopropyl groups of the bis-ketiminate complex $\mathbf{6}$ show two resonances at $\delta 3.30$ and 2.83, while that of the mono-ketiminate complex 7 shows only one resonance at $\delta 3.0$.

Surprisingly, attempts to synthesize cationic aluminum complex by reacting 6 with 1 equiv of $\mathrm{AgBF}_{4}$ in methylene chloride/acetonitrile mix-solvent generates (OCMeCHCMeNAr) $)_{2} \mathrm{AlF}$ (8) in moderate yield (Scheme 3). However, small amounts of unidentified compounds were presented in the product, even after repeated recrystallization. This prevents us from obtaining satisfactory elemental analysis. 
Yu et al.

Table 1. Crystallographic Data and Structure Refinement for Complexes 2-6 and 8

\begin{tabular}{|c|c|c|c|c|c|c|}
\hline & 2 & 3 & 4 & 5 & 6 & 8 \\
\hline empirical formula & $\begin{array}{l}\mathrm{C}_{18} \mathrm{H}_{30} \mathrm{AlNO} \\
315.42\end{array}$ & $\begin{array}{l}\mathrm{C}_{21} \mathrm{H}_{34} \mathrm{AlNO} \\
34347\end{array}$ & $\begin{array}{l}\mathrm{C}_{35} \mathrm{H}_{51} \mathrm{AlN}_{2} \mathrm{O}_{2} \\
558.76\end{array}$ & $\begin{array}{l}\mathrm{C}_{36} \mathrm{H}_{53} \mathrm{AlN}_{2} \mathrm{O}_{2} \\
572.78\end{array}$ & $\begin{array}{l}\mathrm{C}_{34} \mathrm{H}_{48} \mathrm{AlClN}_{2} \mathrm{O}_{2} \\
579.17\end{array}$ & $\begin{array}{l}\mathrm{C}_{34} \mathrm{H}_{48} \mathrm{AlFN}_{2} \mathrm{O}_{2} \\
56272\end{array}$ \\
\hline temp, $\mathrm{K}$ & $\begin{array}{l}315.42 \\
293(2)\end{array}$ & $\begin{array}{l}343.4 / \\
293(2)\end{array}$ & 293(2) & $293(2)$ & $293(2)$ & $293(2)$ \\
\hline wavelength, $\AA$ & 0.71073 & 0.71073 & 0.71073 & 0.71073 & 0.71073 & 0.71073 \\
\hline cryst syst & triclinic & monclinic & triclinic & triclinic & triclinic & monclinic \\
\hline space group & $P \overline{\mathbf{1}}$ & $P 2_{1} / n$ & $P \overline{\mathbf{1}}$ & $P \overline{\mathbf{1}}$ & $P 1$ & $C 2 / c$ \\
\hline \multicolumn{7}{|l|}{ unit cell dimens } \\
\hline$a, \AA$ & $8.9531(12)$ & $10.188(5)$ & $10.4201(12)$ & $10.7606(7)$ & $10.416(3)$ & $24.1082(17)$ \\
\hline$b, \AA$ & $13.5605(17)$ & $20.722(10)$ & $12.3385(15)$ & $12.3944(8)$ & $12.683(4)$ & $11.4039(8)$ \\
\hline$c, \AA$ & $33.193(4)$ & $10.400(5)$ & $14.6368(18)$ & $14.6146(10)$ & $14.364(4)$ & $12.8490(9)$ \\
\hline$\alpha, \operatorname{deg}$ & $89.897(3)$ & 90 & $70.265(2)$ & $70.1030(10)$ & $69.148(6)$ & 90 \\
\hline$\beta, \operatorname{deg}$ & $89.967(3)$ & $95.002(9)$ & $87.053(2)$ & $85.324(2)$ & $87.452(7)$ & $110.0220(10)$ \\
\hline$\gamma, \operatorname{deg}$ & $89.915(2)$ & 90 & $72.735(2)$ & $71.6600(10)$ & $71.598(5)$ & 90 \\
\hline vol, $\AA^{3} / Z$ & $4029.9(9) / 2$ & $2187.2(18) / 4$ & $1689.0(4) / 2$ & $1739.0(2) / 2$ & $1677.6(9) / 1$ & $3319.0(4) / 4$ \\
\hline calcd density, $\mathrm{Mg} / \mathrm{m}^{3}$ & 1.040 & 1.043 & 1.099 & 1.094 & 0.573 & 1.126 \\
\hline abs coeff, $\mathrm{mm}^{-1}$ & 0.103 & 0.099 & 0.091 & 0.090 & 0.085 & 0.097 \\
\hline$F(000)$ & 1376 & 752 & 608 & 624 & 312 & 1216 \\
\hline cryst size, mm & $0.37 \cdot 0.32 \cdot 0.29$ & $0.33 \cdot 0.25 \cdot 0.21$ & $0.38 \cdot 0.35 \cdot 0.29$ & $0.33 \cdot 0.31 \cdot 0.25$ & $0.34 \cdot 0.33 \cdot 0.29$ & $0.32 \cdot 0.28 \cdot 0.23$ \\
\hline$\theta$ for data collection, deg & $1.23-27.55$ & $1.97-27.61$ & $1.48-27.53$ & $1.48-27.56$ & $1.52-27.50$ & $1.80-27.57$ \\
\hline limiting indices & $\begin{array}{l}-11 \leq h \leq+11 \\
-17 \leq k \leq+15 \\
-43 \leq l \leq+37\end{array}$ & $\begin{array}{l}-13 \leq h \leq+12 \\
-26 \leq k \leq+20 \\
-13 \leq l \leq+13\end{array}$ & $\begin{array}{l}-12 \leq h \leq+13 \\
-16 \leq k \leq+14 \\
-19 \leq l \leq+19\end{array}$ & $\begin{array}{l}-14 \leq h \leq+7 \\
-16 \leq k \leq+16 \\
-18 \leq l \leq+18\end{array}$ & $\begin{array}{l}-13 \leq h \leq+11 \\
-16 \leq k \leq+14 \\
-18 \leq l \leq+13\end{array}$ & $\begin{array}{l}-30 \leq h \leq+30 \\
-14 \leq k \leq+14 \\
-12 \leq l \leq+16\end{array}$ \\
\hline no. of reflns collected & 25685 & 13657 & 10761 & 22378 & 10686 & 10345 \\
\hline \multirow[t]{2}{*}{ indep reflns } & 17816 & 4997 & 7475 & 7691 & 8836 & 3796 \\
\hline & $\begin{array}{l}\left(R_{\text {int }}=0.0281\right) \\
\text { empirical used SADABS }\end{array}$ & $\left(R_{\mathrm{int}}=0.0997\right)$ & $\left(R_{\mathrm{int}}=0.0267\right)$ & $\left(R_{\mathrm{int}}=0.0216\right)$ & $\left(R_{\text {int }}=0.0367\right)$ & $\left(R_{\mathrm{int}}=0.0259\right)$ \\
\hline \multicolumn{7}{|l|}{$\begin{array}{l}\text { abs corr } \\
\text { refinement method }\end{array}$} \\
\hline data/restraints /params & $17816 / 0 / 825$ & $4997 / 0 / 225$ & $7475 / 0 / 374$ & $7691 / 0 / 383$ & $8836 / 3 / 745$ & $3796 / 0 / 184$ \\
\hline GOF on $F^{2}$ & 0.544 & 0.710 & 0.593 & 0.885 & 0.511 & 0.781 \\
\hline final $R$ indices & $\mathrm{R} 1=0.0365$ & $\mathrm{R} 1=0.0589$ & $\mathrm{R} 1=0.0371$ & $\mathrm{R} 1=0.0450$ & $\mathrm{R} 1=0.0338$ & $\mathrm{R} 1=0.0373$ \\
\hline$[I>2 \sigma(I)]$ & $w R 2=0.0622$ & $w R 2=0.1148$ & $w R 2=0.0637$ & $w R 2=0.1050$ & $w R 2=0.0565$ & $w R 2=0.0918$ \\
\hline \multirow[t]{2}{*}{$R$ indices (all data) } & $\mathrm{R} 1=0.2101$ & $\mathrm{R} 1=0.1532$ & $\mathrm{R} 1=0.1521$ & $\mathrm{R} 1=0.1138$ & $\mathrm{R} 1=0.1654$ & $\mathrm{R} 1=0.0789$ \\
\hline & $\mathrm{wR} 2=0.0800$ & $\mathrm{wR} 2=0.1373$ & $\mathrm{wR} 2=0.0753$ & $\mathrm{wR} 2=0.1174$ & $\mathrm{wR} 2=0.0752$ & $w R 2=0.1004$ \\
\hline
\end{tabular}

Scheme 3

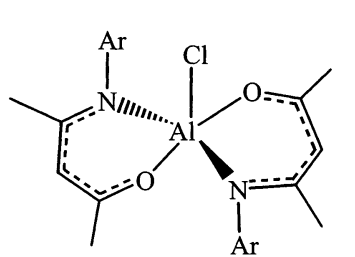

(6)

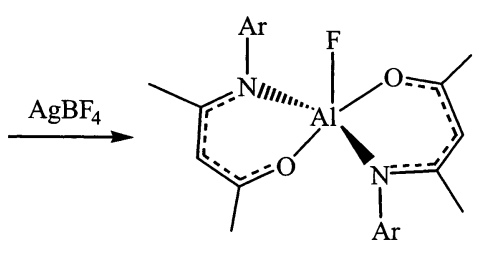

(8)

The ${ }^{1} \mathrm{H}$ and ${ }^{13} \mathrm{C}$ NMR spectra of $\mathbf{8}$ are very similar to that of 6. Structurally characterized aluminum fluoride complexes have been seen; ${ }^{6}$ however, no example of halogen exchange between aluminum chloride and fluoroborate was observed.

Structure of Ketiminate Aluminum Complexes. The structures of complexes $\mathbf{2}-\mathbf{6}$ and $\mathbf{8}$ are determined by X-ray single-crystal analyses. The crystal data and structure refinements of all complexes are summarized in Table 1. Selected bond lengths and angles are listed in Table 2.

Only one of the four independent molecules existing in an unit cell of complex $\mathbf{2}$ is shown, and its ORTEP drawing is displayed in Figure 1. The ORTEP drawing of complex 3

(6) Rennekamp, C.; Wessel, H.; Roesky, H. W.; Muller, P.; Schmidt, H.G.; Noltemeyer, M.; Uson, I.; Barron, A. R. Inorg. Chem. 1999, 38 , 5235. (b) Roesky, H. W.; Stasch, A.; Hatop, H.; Rennekamp, C.; Hamilton, D. H.; Noltemeyer, M.; Schmidt, H.-G. Angew. Chem., Int. Ed. 2000, 39, 171. (c) Hatop, H.; Roesky, H. W.; Labahn, T.; Ropken, C.; Sheldrick, G. M.; Bhattachatjee, M. Organometallics 1998, 17, 4326. (d) Waezsada, S. D.; Feng-Quan Liu.; Barnes, C. E.; Roesky, H. W.; Montero, M. L.; Uson, I. Angew. Chem., Int. Ed. 1997, 36, 2625. (e) Schnitter, C.; Klimek, K.; Roesky, H. W.; Albers, T.; Schmidt, H.-G.; Röpken, C.; Parisini, E. Organometallics 1998, 17, 2249.

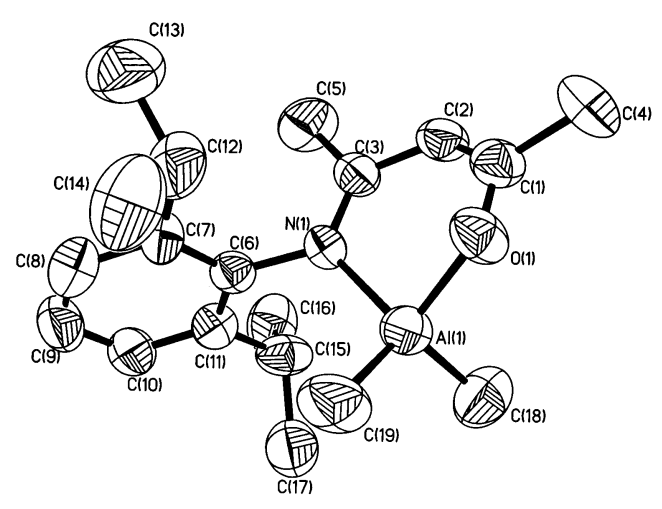

Figure 1. ORTEP drawing of complex 2 with the ellipsoids drawn at $50 \%$ probability. The hydrogen atoms are omitted for clarity.

is shown in Figure 2. Complexes $\mathbf{2}$ and $\mathbf{3}$ both exist as tetrahedron structures with the aluminum atom surrounded by the oxygen and nitrogen atoms of chelating ketiminate and two alkyl groups. The biting angles of ketiminate ligands, $\mathrm{N}(1)-\mathrm{Al}(1)-\mathrm{O}(1)$ (which are $93.9(3)$ and $94.56(9)^{\circ}$ for 2 and $\mathbf{3}$, respectively), are smaller than the regular tetrahedral bond angle of $109.28^{\circ}$. The backbone of ketiminate, $\mathrm{O}(1)-$ $\mathrm{C}(1)-\mathrm{C}(2)-\mathrm{C}(3)-\mathrm{N}(1)$, forms a plane, and the aluminum atom is deviated from the plane at 0.6828 and $0.5035 \AA$ for complexes $\mathbf{2}$ and $\mathbf{3}$, respectively. The bond lengths between atoms of the ketiminate backbone of complexes $\mathbf{2}$ and $\mathbf{3}$ are all in the same ranges, which are comparable to those of similar $\mathrm{Zr}$ and $\mathrm{Ti}$ complexes reported by Smith. ${ }^{4 \mathrm{~d}}$

Complexes 4-6 and 8 exist as five-coordinated trigonal bipyramidal structures, with the two oxygen atoms in the axial positions. There are two independent molecules in a 
Table 2. Selected Bond Lengths $(\AA)$ and Angles (deg) for Complexes $2-6$ and 8

\begin{tabular}{lclr}
\hline \multicolumn{4}{c}{ Compound 2 } \\
$\mathrm{Al}(1)-\mathrm{O}(1)$ & $1.795(5)$ & $\mathrm{Al}(1)-\mathrm{C}(19)$ & $1.7949(6)$ \\
$\mathrm{Al}(1)-\mathrm{C}(18)$ & $1.955(7)$ & $\mathrm{Al}(1)-\mathrm{N}(1)$ & $1.954(6)$ \\
$\mathrm{N}(1)-\mathrm{C}(3)$ & $1.304(8)$ & $\mathrm{C}(2)-\mathrm{C}(3)$ & $1.419(9)$ \\
$\mathrm{C}(2)-\mathrm{C}(1)$ & $1.364(10)$ & $\mathrm{C}(1)-\mathrm{O}(1)$ & $1.302(9)$ \\
$\mathrm{O}(1)-\mathrm{Al}(1)-\mathrm{C}(18)$ & $107.3(3)$ & $\mathrm{O}(1)-\mathrm{Al}(1)-\mathrm{C}(19)$ & $111.6(3)$ \\
$\mathrm{C}(19)-\mathrm{Al}(1)-\mathrm{C}(18)$ & $118.6(4)$ & $\mathrm{O}(1)-\mathrm{Al}(1)-\mathrm{N}(1)$ & $93.9(3)$ \\
$\mathrm{C}(19)-\mathrm{Al}(1)-\mathrm{N}(1)$ & $111.3(3)$ & $\mathrm{C}(18)-\mathrm{Al}(1)-\mathrm{N}(1)$ & $111.3(3)$ \\
\multicolumn{5}{c}{$\mathrm{Compound} \mathbf{3}$} \\
$\mathrm{Al}(1)-\mathrm{O}(1)$ & $1.788(2)$ & $\mathrm{Al}(1)-\mathrm{C}(18)$ & \\
$\mathrm{Al}(1)-\mathrm{C}(20)$ & $1.950(3)$ & $\mathrm{Al}(1)-\mathrm{N}(1)$ & $1.942(3)$ \\
$\mathrm{N}(1)-\mathrm{C}(3)$ & $1.314(3)$ & $\mathrm{C}(2)-\mathrm{C}(3)$ & $1.958(2)$ \\
$\mathrm{C}(2)-\mathrm{C}(1)$ & $1.365(3)$ & $\mathrm{C}(1)-\mathrm{O}(1)$ & $1.323(3)$ \\
\multicolumn{5}{c}{} \\
$\mathrm{O}(1)-\mathrm{Al}(1)-\mathrm{C}(18)$ & $113.27(12)$ & $\mathrm{O}(1)-\mathrm{Al}(1)-\mathrm{C}(20)$ & $105.35(13)$ \\
$\mathrm{C}(18)-\mathrm{Al}(1)-\mathrm{C}(20)$ & $116.15(15)$ & $\mathrm{O}(1)-\mathrm{Al}(1)-\mathrm{N}(1)$ & $94.56(9)$ \\
$\mathrm{C}(18)-\mathrm{Al}(1)-\mathrm{N}(1)$ & $111.17(11)$ & $\mathrm{C}(20)-\mathrm{Al}(1)-\mathrm{N}(1)$ & $114.19(12)$
\end{tabular}

\begin{tabular}{llll}
\multicolumn{5}{c}{ Compound 4} \\
$\mathrm{Al}(1)-\mathrm{O}(1)$ & $1.8202(16)$ & $\mathrm{Al}(1)-\mathrm{O}(2)$ & $1.8076(16)$ \\
$\mathrm{Al}(1)-\mathrm{C}(35)$ & $1.975(2)$ & $\mathrm{Al}(1)-\mathrm{N}(1)$ & $2.0444(17)$ \\
$\mathrm{Al}(1)-\mathrm{N}(2)$ & $2.0518(18)$ & $\mathrm{N}(1)-\mathrm{C}(3)$ & $1.318(2)$ \\
$\mathrm{C}(2)-\mathrm{C}(3)$ & $1.416(3)$ & $\mathrm{C}(2)-\mathrm{C}(1)$ & $1.363(3)$ \\
$\mathrm{C}(1)-\mathrm{O}(1)$ & $1.284(2)$ & $\mathrm{N}(2)-\mathrm{C}(8)$ & $1.316(2)$ \\
$\mathrm{C}(8)-\mathrm{C}(7)$ & $1.407(3)$ & $\mathrm{C}(7)-\mathrm{C}(6)$ & $1.355(3)$ \\
$\mathrm{C}(6)-\mathrm{O}(2)$ & $1.288(2)$ & & \\
& & & \\
$\mathrm{N}(2)-\mathrm{Al}(1)-\mathrm{N}(1)$ & $133.61(7)$ & $\mathrm{C}(35)-\mathrm{Al}(1)-\mathrm{N}(2)$ & $116.31(8)$ \\
$\mathrm{O}(2)-\mathrm{Al}(1)-\mathrm{O}(1)$ & $160.32(8)$ & $\mathrm{C}(35)-\mathrm{Al}(1)-\mathrm{N}(1)$ & $110.05(8)$ \\
$\mathrm{O}(1)-\mathrm{Al}(1)-\mathrm{N}(1)$ & $87.75(7)$ & $\mathrm{O}(2)-\mathrm{Al}(1)-\mathrm{N}(2)$ & $87.41(7)$
\end{tabular}

\begin{tabular}{llll}
\multicolumn{5}{c}{ Compound $\mathbf{5}$} \\
$\mathrm{Al}(1)-\mathrm{O}(1)$ & $1.8098(13)$ & $\mathrm{Al}(1)-\mathrm{O}(2)$ & $1.8181(14)$ \\
$\mathrm{Al}(1)-\mathrm{C}(35)$ & $1.957(2)$ & $\mathrm{Al}(1)-\mathrm{N}(1)$ & $2.0616(18)$ \\
$\mathrm{Al}(1)-\mathrm{N}(2)$ & $2.0678(17)$ & $\mathrm{N}(1)-\mathrm{C}(3)$ & $1.325(2)$ \\
$\mathrm{C}(2)-\mathrm{C}(3)$ & $1.412(3)$ & $\mathrm{C}(2)-\mathrm{C}(1)$ & $1.352(3)$ \\
$\mathrm{C}(1)-\mathrm{O}(1)$ & $1.287(3)$ & $\mathrm{O}(2)-\mathrm{C}(18)$ & $1.290(2)$ \\
$\mathrm{C}(18)-\mathrm{C}(19)$ & $1.351(3)$ & $\mathrm{C}(19)-\mathrm{C}(20)$ & $1.414(2)$ \\
$\mathrm{N}(2)-\mathrm{C}(20)$ & $1.326(2)$ & & \\
& & & \\
$\mathrm{N}(2)-\mathrm{Al}(1)-\mathrm{N}(1)$ & $138.25(7)$ & $\mathrm{C}(35)-\mathrm{Al}(1)-\mathrm{N}(2)$ & $111.24(9)$ \\
$\mathrm{O}(2)-\mathrm{Al}(1)-\mathrm{O}(1)$ & $155.97(8)$ & $\mathrm{C}(35)-\mathrm{Al}(1)-\mathrm{N}(1)$ & $110.51(9)$ \\
$\mathrm{O}(1)-\mathrm{Al}(1)-\mathrm{N}(1)$ & $87.35(7)$ & $\mathrm{O}(2)-\mathrm{Al}(1)-\mathrm{N}(2)$ & $87.52(7)$
\end{tabular}

\begin{tabular}{lrll}
\multicolumn{5}{c}{ Compound 6} \\
$\mathrm{Al}(1)-\mathrm{O}(1)$ & $1.805(9)$ & $\mathrm{Al}(1)-\mathrm{O}(2)$ & $1.799(9)$ \\
$\mathrm{Al}(1)-\mathrm{Cl}(1)$ & $2.170(6)$ & $\mathrm{Al}(1)-\mathrm{N}(1)$ & $2.021(12)$ \\
$\mathrm{Al}(1)-\mathrm{N}(2)$ & $2.035(12)$ & $\mathrm{N}(1)-\mathrm{C}(14)$ & $1.346(14)$ \\
$\mathrm{C}(14)-\mathrm{C}(15)$ & $1.374(18)$ & $\mathrm{C}(15)-\mathrm{C}(16)$ & $1.32(2)$ \\
$\mathrm{C}(16)-\mathrm{O}(1)$ & $1.281(15)$ & $\mathrm{O}(2)-\mathrm{C}(33)$ & $1.290(14)$ \\
$\mathrm{C}(33)-\mathrm{C}(32)$ & $1.375(18)$ & $\mathrm{C}(31)-\mathrm{C}(32)$ & $1.448(15)$ \\
$\mathrm{N}(2)-\mathrm{C}(31)$ & $1.358(13)$ & & \\
& & & \\
$\mathrm{N}(2)-\mathrm{Al}(1)-\mathrm{N}(1)$ & $134.6(4)$ & $\mathrm{Cl}(1)-\mathrm{Al}(1)-\mathrm{N}(2)$ & $108.2(4)$ \\
$\mathrm{O}(2)-\mathrm{Al}(1)-\mathrm{O}(1)$ & $168.8(5)$ & $\mathrm{Cl}(1)-\mathrm{Al}(1)-\mathrm{N}(1)$ & $117.2(3)$ \\
$\mathrm{O}(1)-\mathrm{Al}(1)-\mathrm{N}(1)$ & $88.6(5)$ & $\mathrm{O}(2)-\mathrm{Al}(1)-\mathrm{N}(2)$ & $91.2(5)$
\end{tabular}

\begin{tabular}{llll}
\multicolumn{5}{c}{ Compound 8} \\
$\mathrm{Al}(1)-\mathrm{O}(1)$ & $1.804(1)$ & $\mathrm{Al}(1)-\mathrm{O}(1 \mathrm{~A})$ & $1.804(1)$ \\
$\mathrm{Al}(1)-\mathrm{F}(1)$ & $1.6775(14)$ & $\mathrm{Al}(1)-\mathrm{N}(1)$ & $1.9984(12)$ \\
$\mathrm{Al}(1)-\mathrm{N}(1 \mathrm{~A})$ & $1.9984(12)$ & $\mathrm{N}(1)-\mathrm{C}(3)$ & $1.329(2)$ \\
$\mathrm{N}(1)-\mathrm{C}(3)$ & $1.329(2)$ & $\mathrm{C}(1)-\mathrm{C}(5 \mathrm{~A})$ & $1.357(2)$ \\
$\mathrm{O}(1)-\mathrm{C}(1)$ & $1.287(2)$ & $\mathrm{C}(3)-\mathrm{C}(5)$ & $1.410(2)$ \\
$\mathrm{C}(5)-\mathrm{C}(1 \mathrm{~A})$ & $1.357(2)$ & & \\
$\mathrm{N}(1)-\mathrm{Al}(1)-\mathrm{N}(1 \mathrm{~A})$ & $134.49(7)$ & $\mathrm{F}(1)-\mathrm{Al}(1)-\mathrm{N}(1)$ & $112.76(4)$ \\
$\mathrm{O}(1)-\mathrm{Al}(1)-\mathrm{O}(1 \mathrm{~A})$ & $169.64(8)$ & $\mathrm{F}(1)-\mathrm{Al}(1)-\mathrm{N}(1 \mathrm{~A})$ & $112.76(4)$ \\
$\mathrm{O}(1)-\mathrm{Al}(1)-\mathrm{N}(1 \mathrm{~A})$ & $89.22(5)$ & $\mathrm{O}(1 \mathrm{~A})-\mathrm{Al}(1)-\mathrm{N}(1)$ & $89.22(5)$
\end{tabular}

unit cell for complex $\mathbf{6}$; however, only one is described due to their similarity. The ORTEP drawings of complex 4-6 and 8 are shown in Figures 3-6. The bond lengths of aluminum atom to oxygen and nitrogen atoms in complexes 4-6 and 8 are all in the ranges of 1.80 and $2.00 \AA$, respectively. Moreover, the total angles of the trigonal plane for all the complexes are close to $360^{\circ}$. However, in

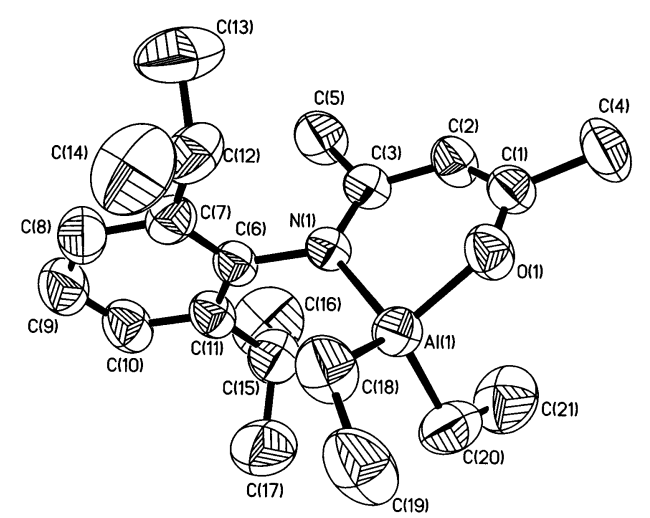

Figure 2. ORTEP drawing of complex 3 with the ellipsoids drawn at $50 \%$ probability. The hydrogen atoms are omitted for clarity.

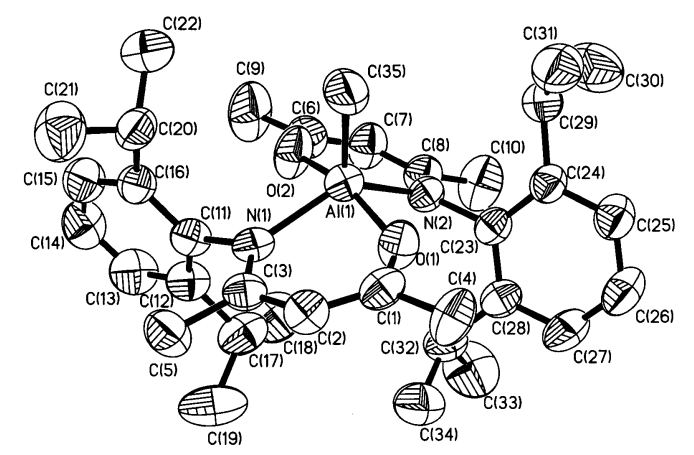

Figure 3. ORTEP drawing of complex 4 with the ellipsoids drawn at $50 \%$ probability. The hydrogen atoms are omitted for clarity.

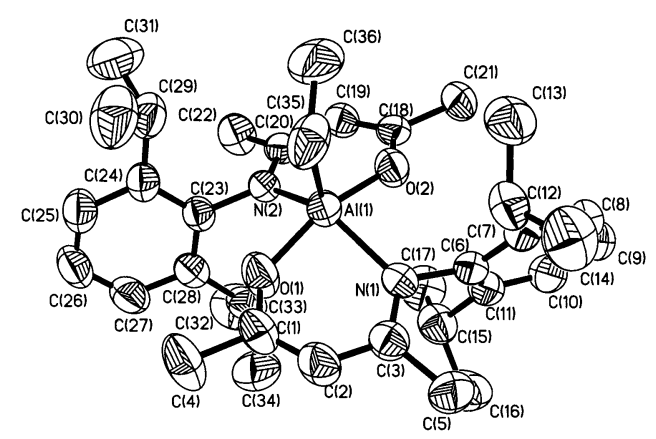

Figure 4. ORTEP drawing of complex 5 with the ellipsoids drawn at $50 \%$ probability. The hydrogen atoms are omitted for clarity.

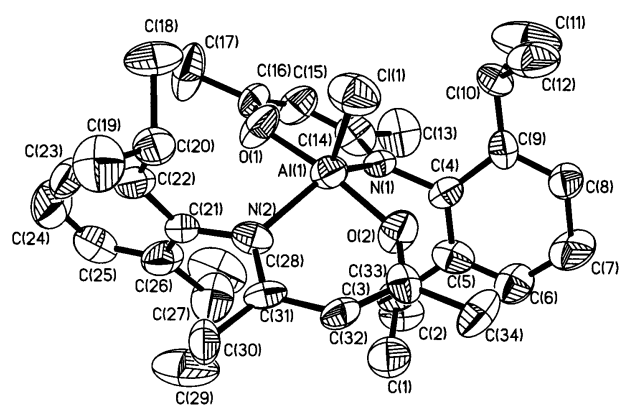

Figure 5. ORTEP drawing of complex 6 with the ellipsoids drawn at $50 \%$ probability. The hydrogen atoms are omitted for clarity.

comparing the angles of axial atoms, the more sterically crowded ethyl group bends the angle of $\mathrm{O}(2)-\mathrm{Al}(1)-\mathrm{O}(1)$ of complex 5 away from linear to $155.97(8)^{\circ}$, while the less hindered fluorine atoms keeps the angle of $\mathrm{O}(1 \mathrm{~A})-\mathrm{Al}(1)-$ $\mathrm{O}(1)$ of complex 8 closer to linearity at $169.64(5)^{\circ}$. Again, 
Yu et al.

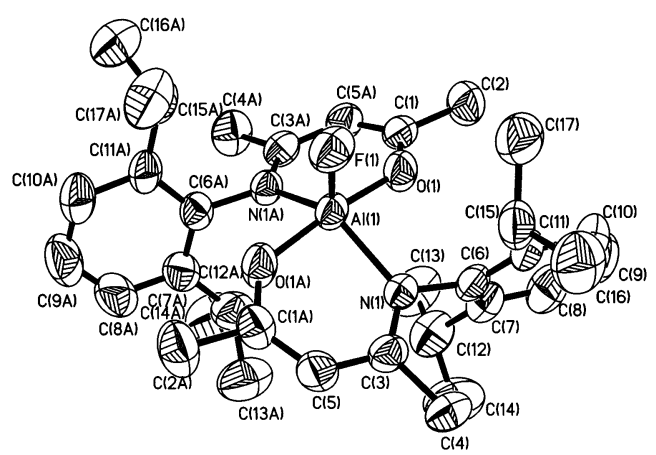

Figure 6. ORTEP drawing of complex 8 with the ellipsoids drawn at $50 \%$ probability. The hydrogen atoms are omitted for clarity.

Table 3. Ring-Opening Polymerization of $\epsilon$-Caprolactone Catalyzed by Aluminum Ketiminate Complexes ${ }^{a}$

\begin{tabular}{rccccrrr}
\hline entry & catalyst & monomer $(\mathrm{g})$ & time $(\mathrm{h})$ & temp $\left({ }^{\circ} \mathrm{C}\right)$ & \multicolumn{1}{c}{$\mathrm{M}_{\mathrm{n}}$} & PDI & yield \\
\hline 1 & $\mathbf{2}$ & 3.26 & 3.5 & 60 & 34997 & 1.99 & 93.7 \\
2 & $\mathbf{2}$ & 3.26 & 2.4 & 25 & 25210 & 1.64 & 93.7 \\
3 & $\mathbf{3}$ & 2.99 & 3.5 & 60 & 100362 & 1.75 & 92.1 \\
4 & $\mathbf{3}$ & 2.99 & 2.4 & 25 & 26560 & 2.80 & 91.7 \\
5 & $\mathbf{4}$ & 1.84 & 3.5 & 60 & 44009 & 2.35 & 94.3 \\
6 & $\mathbf{4}$ & 1.84 & 2.4 & 25 & 3717 & 4.65 & 90.8 \\
7 & $\mathbf{5}$ & 1.80 & 3.5 & 60 & 77597 & 2.06 & 96.1 \\
8 & $\mathbf{5}$ & 1.80 & 2.4 & 25 & 37210 & 1.79 & 95.8 \\
9 & $\mathbf{6}$ & 1.77 & 3.5 & 60 & 778 & 1.34 & 95.6 \\
10 & $\mathbf{6}$ & 1.77 & 2.4 & 25 & 746 & 1.29 & 91.5
\end{tabular}

${ }^{a}$ Reaction conditions: $2 \mathrm{~mL}$ of toluene was used as solvent; $0.03 \mathrm{~g}$ of catalyst was used for the polymerization; 300 equiv of monomer was used.

the bond lengths of ketiminate backbone of complexes 4-6 and $\mathbf{8}$ are comparable with those in complexes $\mathbf{2}$ and $\mathbf{3}$.

Ring Opening Polymerization of $\epsilon$-Caprolactone. Ringopening polymerizations of $\epsilon$-caprolactone by aluminum ${ }^{7}$ and other metal complexes ${ }^{8}$ have been seen. The ring-opening polymerization of $\epsilon$-caprolactone catalyzed by complexes 2-6 have been examined. In general, the $\epsilon$-caprolactone and catalysts were placed in Schlenk flask separately and toluene ( $2 \mathrm{~mL}$ ) was filled. The monomer was added to the catalyst/ toluene solution and stirred at room temperature or $60{ }^{\circ} \mathrm{C}$. The results are listed in Table 3. Mono- and bis-ketiminate aluminum alkyl complexes all show activities toward the polymerization of $\epsilon$-caprolactone. However, the corresponding aluminum chloride complex only results in oligomer. In general, the higher temperature results in higher poly(caprolactone) for all catalysts (entries 1-8) excepting 6 (entries 9-10). In the case of entry $3, M_{n}$ reaches to $10^{5}$. The PDI values are generally low, which are comparable with the diketiminate aluminum system by $\mathrm{Chen}^{7 \mathrm{~g}}$ but are larger than aluminum ${ }^{7 f}$ and yittrium ${ }^{8 \mathrm{a}}$ alkoxide systems. The

(7) Huang, C.-H.; Wang, F.-C.; Ko, B.-T.; Yu, T.-L.; Lin, C.-C. Macromolecules 2001, 34, 356. (b) Wu, B.; Lenz, R. W.; Hazer, B. Macromolecules 1999, 32, 6856. (c) Tian, D.; Dubois, P.; Jérôme, R. Macromolecules 1997, 30, 2575. (d) Duda, A. Macromolecules 1996, 29, 1399. (e) Chen, H.-L.; Ko, B.-T.; Huang, B.-H.; Lin, C.-C. Organometallics 2001, 20, 5076. (f) Liu, Y.-C.; Ko, B.-T.; Lin, C.-C. Macromolecules 2001, 34, 6196. (g) Chakraborty, D.; Chen, E. Y.-X. Organometallics 2002, 21, 1438.

(8) Chamberlain, B. M.; Jazdzewski, B.; Pink, M.; Hillmyer, M. A.; Tolman, W. B. Macromolecules 2000, 33, 3970. (b) Ravi, P.; Gröb, T.; Dehnicke, K.; Greineer, A. Macromolecules 2001, 34, 8649. (c) Martin, E.; Dubois, P.; Jérôme, R. Macromolecules 2000, 33, 1530. (d) Takeuchi, D.; Nakamura, T.; Aida, T. Macromolecules 2000, 33, 725. (e) Stevels, W. M.; Ankoné, M. J. K.; Dijkstra, P.; Feijen, J. Macromolecules 1996, 29, 8296.
Scheme 4

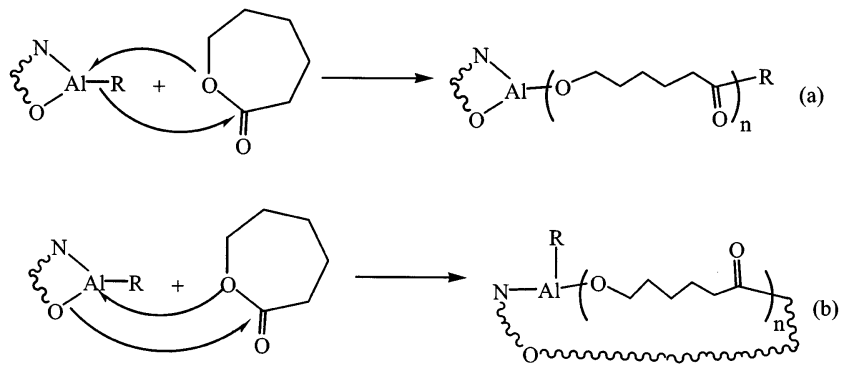

results also indicate that the catalysts serve as single site catalysts for the ring-opening polymerization of $\epsilon$-caprolactone. However, by conversion of complexes 2-6 to aluminum alkoxide complexes may be a trend for obtaining a polymer with narrower PDI.

A possible polymerization mechanism for the ring-opening polymerization is shown in Scheme 4a. An anionic coordination mechanism for the ring-opening polymerization of $\epsilon$-caprolactone can be observed from the reactions of entries 1-8 and entries 9-10. While the more reactive $\mathrm{Al}-$ alkyl complexes were used as catalysts, the reactions were faster and higher molecular weight polymers resulted. On the other hand, while the Al-chloride complex was used as catalyst, a low molecular weight oligomer was obtained, indicating the inert $\mathrm{Al}$-chloride bond. However, we are not able to eliminate the possibility of initiation occurring at the Alketiminate bond, as shown in Scheme 4b. A similar polymerization mechanism has been proposed by Chen. ${ }^{7 \mathrm{~g}}$ That is observed from the interesting results in which the polymer molecular weight increased sharply from the methyl to ethyl derivatives (entry 3 vs 1 ; entry 7 vs 5 ). One possible reason is the bulkier ethyl group protecting the active species from aggregation, which results in a higher polymer. A more detailed mechanism is under investigation.

\section{Concluding Remarks}

We have been successful in the synthesis of a series of aluminum complexes containing ketiminate ligands. All the complexes are found to be active toward the ring-opening polymerization of $\epsilon$-caprolactone. The aluminum alkyl complexes are generally causing a higher molecular weight polymer, while the aluminum halide complex only results in low molecular weight oligomer. A mechanistic study of the ring-opening polymerization as well as the steric effects of the substitutents of the aluminum alkyl groups is our current investigation.

\section{Experimental Section}

General Procedures. All reactions were performed under a dry nitrogen atmosphere using standard Schlenk line or glovebox technique. Toluene and diethyl ether were dried by refluxing over sodium benzophenone ketyl. $\mathrm{CH}_{2} \mathrm{Cl}_{2}$ and acetonitrile were dried over $\mathrm{P}_{2} \mathrm{O}_{5}$ and $\mathrm{CaH}_{2}$, respectively. All solvents were distilled and stored in solvent reservoirs, which contained $4 \AA$ molecular sieves, and purged with nitrogen. ${ }^{1} \mathrm{H}$ and ${ }^{13} \mathrm{C}$ NMR spectra were recorded on a Bruker AC200 spectrometer. Chemical shifts for ${ }^{1} \mathrm{H}$ and ${ }^{13} \mathrm{C}$ spectra were recorded in parts per million relative to the residual proton of $\mathrm{CDCl}_{3}(\delta 7.24)$. Elemental analyses were performed on 
a Heraeus $\mathrm{CHN}-\mathrm{OS}$ Rapid Elemental Analyzer at Instrument Center, NCHU. Ketimine (1), OCMeCHCMeNHAr where $\mathrm{Ar}=$ 2,6-diisopropylphenyl, was prepared according to previously reported procedure. ${ }^{3 \mathrm{a}} \mathrm{AlCl}_{3}$ (Strem), MeLi, and EtLi (Aldrich) were used as received.

(OCMeCHCMeNAr)AlMe2 (2). $\mathrm{AlMe}_{3}(1.9 \mathrm{~mL}, 2 \mathrm{M}, 3.80$ mmol) was added to a stirred solution of $\mathbf{1}(1.0 \mathrm{~g}, 3.86 \mathrm{mmol})$ in $20 \mathrm{~mL}$ of toluene at $0{ }^{\circ} \mathrm{C}$. The mixture was then allowed to warm to room temperature and stirred for additional $3 \mathrm{~h}$. The mixture was vacuum-dried to yield a pale yellow oil, which was solidified after being immersed in liquid nitrogen and warmed to room temperature. The solid was recrystallized from either toluene or heptane at $-20{ }^{\circ} \mathrm{C}$ to yield $1.10 \mathrm{~g}$ colorless product ( $92 \%$ yield). ${ }^{1} \mathrm{H} \mathrm{NMR}\left(\mathrm{CDCl}_{3}\right): \delta-0.93$ (s, 6H, AlMe $\left.e_{2}\right), 1.08(\mathrm{~d}, 6 \mathrm{H}, \mathrm{CHMe} 2)$, 1.19 (d, 6H, CHMe $), 1.74$ (s, 3H, CMe), 2.07 (s, 3H, CMe), 2.92 (m, 2H, CHMe $), 5.34$ (s, 1H, CMeCHCMe), 7.20 (m, 3H, Ph). ${ }^{13} \mathrm{C} \mathrm{NMR}\left(\mathrm{CDCl}_{3}\right): \delta-11.0,23.2,24.4,24.6,25.8,27.9,100.4$, 124.2, 127.2, 139.0, 142.9, 176.1, 181.0. Anal. Calcd for $\mathrm{C}_{19} \mathrm{H}_{30^{-}}$ NOAl: C, 72.35; H, 9.59. Found: C, 72.31; H, 9.82.

(OCMeCHCMeNAr)AlEt2 (3). A similar manner was taken as that for preparing complex $\mathbf{2}$. Complex $\mathbf{3}$ was prepared from $\mathrm{AlEt}_{3}(5.1 \mathrm{~mL}, 1.9 \mathrm{M}, 9.69 \mathrm{mmol})$ and 1 (2.5 g, $\left.9.65 \mathrm{mmol}\right)$ in $98 \%$ yield as a pale yellow oil, which partially solidified on standing over a period of time in a glovebox. ${ }^{1} \mathrm{H} \mathrm{NMR}\left(\mathrm{CDCl}_{3}\right): \delta-0.22$ (m, $4 \mathrm{H}, \mathrm{AlCH}_{2} \mathrm{CH}_{3}$ ), 0.86 (t, $\left.6 \mathrm{H}, \mathrm{AlCH}_{2} \mathrm{CH}_{3}\right), 1.10$ (d, 6H, $\mathrm{CHMe}$ ), 1.22 (d, 6H, CHMe $e_{2}$, 1.75 (s, 3H, CMe), 2.10 (s, 3H, CMe), 2.93 (m, 2H, CHMe $)$ ) 5.34 (s, 1H, CMeCHCMe), 7.20 (m, 3H, Ph). ${ }^{13} \mathrm{C} \mathrm{NMR}\left(\mathrm{CDCl}_{3}\right): \delta-0.9,8.9,23.1,24.3,24.7,25.7,27.9,100.6$, 124.2, 127.2, 139.3, 142.9, 176.3, 181.4 .

(OCMeCHCMeNAr) 2AIMe (4). A similar manner was taken as that for preparing complex $\mathbf{2}$; however, longer reaction time was needed. Complex 4 was prepared by stirring $\mathrm{AlMe}_{3}(4.8 \mathrm{~mL}, 2.0$ $\mathrm{M}, 9.6 \mathrm{mmol})$ and $1(5.0 \mathrm{~g}, 19.31 \mathrm{mmol})$ in toluene at $100{ }^{\circ} \mathrm{C}$ for 3 days. Colorless crystals were obtained in $78 \%$ yield after recrystallization from toluene at $-20{ }^{\circ} \mathrm{C}(4.18 \mathrm{~g}) .{ }^{1} \mathrm{H}$ NMR $\left(\mathrm{CDCl}_{3}\right): \delta-0.97$ (s, 3H, $\left.\mathrm{AlCH}_{3}\right), 1.12\left(\mathrm{~m}, 24 \mathrm{H}, \mathrm{CHMe} e_{2}\right), 1.17$ (s, 6H, CMe), 1.61 (s, 6H, CMe), 2.93 (m, 2H, CHMe $), 3.06$ (m, $2 \mathrm{H}, \mathrm{CHMe}$ ), 5.06 (s, 2H, CMeCHCMe), $7.10(\mathrm{~m}, 6 \mathrm{H}, \mathrm{Ph}) \cdot{ }^{13} \mathrm{C}$ NMR $\left(\mathrm{CDCl}_{3}\right): \delta-10.5,23.6,23.7,24.3$ (br, two peaks overlapping), 24.9, 25.4, 27.5, 28.3, 99.2, 122.9, 123.3, 124.7, 141.7, 146.1, 174.1, 177.5. Anal. Calcd for $\mathrm{C}_{35} \mathrm{H}_{51} \mathrm{~N}_{2} \mathrm{O}_{2} \mathrm{Al}$ : C, 75.23; $\mathrm{H}$, 9.20; N, 5.01. Found: C,75.75; H, 9.39; N, 5.69.

(OCMeCHCMeNAr) 2AlEt (5). A similar manner as that for preparing complex 2 was adopted. However, longer reaction time was needed. Complex 5 was prepared in $73 \%$ yield by stirring $\mathrm{AlEt}_{3}$ $(5.1 \mathrm{~mL}, 1.9 \mathrm{M}, 9.69 \mathrm{mmol})$ and $\mathbf{1}(5.0 \mathrm{~g}, 19.31 \mathrm{mmol})$ in toluene at $100{ }^{\circ} \mathrm{C}$ for 3 days. Colorless crystals were obtained after recrystallization from toluene at $-20{ }^{\circ} \mathrm{C}(4.01 \mathrm{~g}) .{ }^{1} \mathrm{H}$ NMR $\left(\mathrm{CDCl}_{3}\right): \delta-0.27\left(\mathrm{~m}, 1 \mathrm{H}, \mathrm{AlCH}_{a} \mathrm{H}_{\mathrm{b}} \mathrm{CH}_{3}\right),-0.08\left(\mathrm{~m}, 1 \mathrm{H}, \mathrm{AlCH}_{\mathrm{a}} H_{\mathrm{b}^{-}}\right.$ $\left.\mathrm{CH}_{3}\right), 0.89$ (t, 3H, $\left.\mathrm{AlCH}_{2} \mathrm{CH}_{3}\right), 1.06-1.28$ (m, 24H, CHMe $\left.e_{2}\right), 1.17$ (s, 6H, CMe), 1.62 (s, 6H, CMe), 2.94 (m, 2H, CHMe $), 3.19$ (m, $2 \mathrm{H}, \mathrm{CHMe}$ ), 5.00 (s, 2H, CMeCHCMe), 7.12 (m, 6H, Ph). ${ }^{13} \mathrm{C}$ $\mathrm{NMR}\left(\mathrm{CDCl}_{3}\right): \delta 3.4,10.2,23.7,23.8,24.5,24.8,25.0,27.6,28.1$, 28.5, 98.9, 122.9, 123.3, 124.8, 141.3, 142.2, 146.4, 175.2, 178.6. Anal. Calcd for $\mathrm{C}_{36} \mathrm{H}_{53} \mathrm{~N}_{2} \mathrm{O}_{2} \mathrm{Al}$ : C, 75.49; H, 9.33; N, 4.89. Found: C, 75.25; H, 9.93; N, 5.46.

(OCMeCHCMeNAr) 2AICl (6). A toluene solution $(30 \mathrm{~mL})$ of lithiated $1(8.0 \mathrm{~g}, 30 \mathrm{mmol})$ was added to a $\mathrm{AlCl}_{3}(2.0 \mathrm{~g}, 15$ mmol) and toluene $(20 \mathrm{~mL})$ suspension at $0{ }^{\circ} \mathrm{C}$ with stirring. The suspension was warmed to room temperature and stirred for an additional $3 \mathrm{~h}$. The mixture was filtered through Celite to remove $\mathrm{LiCl}$, and the filtrate was dried under vacuum to yield an off-white solid, which was recrystallized from toluene at $-20{ }^{\circ} \mathrm{C}$ to generate $8.7 \mathrm{~g}$ (78\% yield) of colorless crystalline product. ${ }^{1} \mathrm{H}$ NMR $\left(\mathrm{CDCl}_{3}\right): \delta 1.04-1.25\left(\mathrm{~m}, 24 \mathrm{H}, \mathrm{CHMe} e_{2}\right), 1.21(\mathrm{~s}, 6 \mathrm{H}, \mathrm{CMe}), 1.68$ (s, 6H, CMe), $2.82(\mathrm{~m}, 2 \mathrm{H}, \mathrm{CHMe}$ ), 3.30 (m, 2H, CHMe $), 5.22$ (s, $2 \mathrm{H}, \mathrm{CMeCHCMe}), 7.15(\mathrm{~m}, 6 \mathrm{H}, \mathrm{Ph}) .{ }^{13} \mathrm{C} \mathrm{NMR}\left(\mathrm{CDCl}_{3}\right): \delta$ 23.5, 23.6, 24.4, 24.8 (br, two peaks overlapping), 25.8, 27.6, 28.4, 100.3, 122.8, 124.0, 125.3, 141.0, 142.8, 145.2, 176.6, 178.5. Anal. Calcd for $\mathrm{C}_{34} \mathrm{H}_{48} \mathrm{~N}_{2} \mathrm{O}_{2} \mathrm{ClAl}$ : C, 70.51; H, 8.35; N, 4.84. Found: C, 69.93; H, 8.19; N, 5.67.

(OCMeCHCMeNAr) AlCl2 (7). A similar manner as that for preparing complex $\mathbf{6}$ was taken. Complex 7 was prepared from $\mathrm{AlCl}_{3}(0.5 \mathrm{~g}, 3.76 \mathrm{mmol})$ and lithiated $1(1.0 \mathrm{~g}, 3.77 \mathrm{mmol})$, and $0.78 \mathrm{~g}(58 \%)$ of the desired product was obtained with a small amount of impurity presented. ${ }^{1} \mathrm{H}$ NMR $\left(\mathrm{CDCl}_{3}\right): \delta 1.08-1.29$ (m, 12H, CHMe $), 1.88$ (s, 3H, CMe), 2.18 (s, 3H, CMe), 2.94 (m, 2H, $\mathrm{CHMe}$ ), 5.61 (s, 1H, CMeCHCMe), $7.27(\mathrm{~m}, 6 \mathrm{H}, \mathrm{Ph}) .{ }^{13} \mathrm{C}$ $\mathrm{NMR}\left(\mathrm{CDCl}_{3}\right): \delta 23.8,24.4,24.9,25.5,28.3,102.0,124.8,128.5$, 136.0, 143.5, 180.8, 182.3.

(OCMeCHCMeNAr) 2AIF (8). A Schlenk flask was charged $6(0.30 \mathrm{~g}, 0.52 \mathrm{mmol})$ and $\mathrm{AgBF}_{4}(0.10 \mathrm{~g}, 0.51 \mathrm{mmol})$ in a glovebox. Methylene chloride $(10 \mathrm{~mL})$ and acetonitrile $(10 \mathrm{~mL})$ were added to 6 and $\mathrm{AgBF}_{4}$, respectively. The $\mathrm{AgBF}_{4}$ solution was added dropwise to the methylene chloride solution at $0{ }^{\circ} \mathrm{C}$ and stirred for another hour at room temperature. The solvent was removed, and the residual was extracted with methylene chloride and filtered through Celite. The filtrate was concentrated to a small amount of volume. Colorless crystals were obtained from the concentrated methylene chloride solution at $-20{ }^{\circ} \mathrm{C}$. A $0.29 \mathrm{~g}$ amount of solid was obtained $(76 \%$ yield). Small amounts of unidentified compounds were present in the product, even after repeated recrystallization. ${ }^{1} \mathrm{H}$ NMR $\left(\mathrm{CDCl}_{3}\right): \delta 1.04-1.25(\mathrm{~m}, 24 \mathrm{H}$, $\mathrm{CHMe}_{2}$ ), 1.21 (s, 6H, CMe), 1.63 (s, 6H, CMe), 2.77 (m, 2H, $\mathrm{CHMe}$ ), 3.18 (m, 2H, CHMe $), 5.15$ (s, 2H, CMeCHCMe), 7.15 $(\mathrm{m}, 6 \mathrm{H}, \mathrm{Ph})$.

$\epsilon$-Caprolactone Polymerization. A general procedure for $\epsilon$-caprolactone polymerization catalyzed by aluminum complexes was described as follow. In a glovebox, $\epsilon$-caprolactone and catalyst were placed in separate Schlenk flasks and moved out of the glovebox. Toluene $(2 \mathrm{~mL})$ was added with a syringe to the catalyst and heated to the desired temperature followed by the addition of $\epsilon$-caprolactone. The reactions were proceeded in desired condition and quenched with water. Solid was obtained by filtration, washed with methanol to remove excess metal catalysts, and dried under vacuum.

$\mathrm{X}$-ray Structure Determination of Complexes 2-6 and 8. Crystals of complex $\mathbf{2}$ were obtained from a concentrated heptane solution of $\mathbf{1}$ at $-20{ }^{\circ} \mathrm{C}$. Crystals of $\mathbf{3}$ were obtained directly from the sublimation of viscous complex $\mathbf{3}$ by storing it in a glovebox. Crystals of 4-6 were obtained from saturated toluene solution at $-20{ }^{\circ} \mathrm{C}$, and crystals of $\mathbf{8}$ were obtained from a concentrated methylene chloride solution of $\mathbf{8}$. All of the crystals were sealed in a glass capillary and mounted on a goniostat. Data collections were proceeded at 293(2) K for all complexes. Data were collected on a Bruker SMART CCD diffractometer with graphite-monochromated Mo K $\alpha$ radiation. Structural determinations were made using the SHELXTL package of programs. All refinements were carried out by full-matrix least squares using anisotropic displacement parameters for all non-hydrogen atoms. All the hydrogen atoms are calculated. The crystal data are summarized in Table 1.

Acknowledgment. We thank the National Science Council of Taiwan for financial support and the National Center for High Performance Computing for databank search.

Supporting Information Available: Single-crystal X-ray structure data of complexes $\mathbf{2}-\mathbf{6}$ and $\mathbf{8}$ (CIF format). This material is available free of charge via the Internet at http://pubs.acs.org.

IC025785J 